\title{
Declaración de Salamanca: Avances y Fisuras desde las ONGs de/para Personas con Discapacidad
}

\author{
Salamanca Statement: Progress and Fissures from NGOs of/for \\ People with Disabilities
}

\author{
$\mathrm{M}^{\mathrm{a}}$ Eugenia Yadarola * \\ FUSDAI y Universidad Católica de Córdoba, Argentina
}

\begin{abstract}
En este artículo se retoma aquellos avances en la "Declaración de Salamanca y Marco de Acción para las necesidades educativas especiales" que significaron una herramienta importante para las Organizaciones no Gubernamentales de y para las personas con discapacidad y sus familias - ONGS/PCDF- en la defensa por una inclusión educativa para las personas con discapacidad. De allí, a la luz de la evolución del enfoque del derecho a la inclusión, analizar la Declaración de Salamanca y desentrañar algunas fisuras que su letra dejó entrever, entendidas como debilidades que pudieron filtrarse en políticas y prácticas limitadas desde un enfoque integrador y más centrado en el déficit de las personas con discapacidad. Fisuras observadas en políticas y prácticas en Argentina como en otros países que, sumados a otros factores, pudieron convertirse en barreras para la inclusión educativa real y de calidad de los/as niños/as y jóvenes con discapacidad, quitando fuerzas para su defensa desde las ONGS/PCDF. Por ello, es fundamental que las ONGS/PCDF participen activamente y sean realmente inclusivas en sus políticas y prácticas para que de modo coherente y decisivo puedan lograr incidir en la construcción de sistemas educativos inclusivos y sociedades más inclusivas. Asimismo, el trabajo en redes resulta clave.
\end{abstract}

Descriptores: Inclusión educativa; Personas con discapacidad; Organización no gubernamental; Política educacional; Oportunidades educativas.

This article recalls those advances in the "Salamanca Statement and Framework for Action for special educational needs" that meant an important tool for nongovernmental organizations of and for people with disabilities and their families NGOS/PWDF- in the advocacy for educational inclusion for people with disabilities. From there, in light of the evolution of the approach to the right to inclusion, analyze the Salamanca Statament and unravel some cracks that its letter showed, understood as weaknesses that could seep into limited policies and practices from an integrative and more focused approach in the deficit of people with disabilities. Fissures observed in policies and practices in Argentina as in other countries that, in addition to other factors, could become barriers to the real and quality educational inclusion of children and young people with disabilities, removing forces for their defense from the NGOS/PWDF. Therefore, it is essential that NGOS/PWDF actively participate and be truly inclusive in their policies and practices so that in a coherent and decisive way they can influence the construction of inclusive education systems and more inclusive societies. Also, networking is key.

Keywords: Educational inclusion; People with disabilities; Nongovernmental organizations; Educational policy; Educational opportunities.

*Contacto: m.eugenia.yadarola@gmail.com 


\section{Introducción}

La "Declaración de Salamanca y Marco de Acción para las necesidades educativas especiales" (UNESCO, 1994) fue un hito fundamental en la defensa de una educación inclusiva de calidad para la diversidad de estudiantes y particularmente para las personas con discapacidad. Posteriormente, se consolida y evoluciona luego en la Convención de los Derechos de las Personas con Discapacidad-CDPD- (ONU, 2006).

Para las Organizaciones no Gubernamentales de y para las personas con discapacidad y sus familias -ONGS/PCDF- la Declaración de Salamanca (que llamaré Declaración), significó tanto una orientación a conquistar, como una herramienta en defensa de una educación integradora, que perfilaba como inclusiva. La Declaración insta a los gobiernos a "fomentar y facilitar la participación de padres, comunidades y organizaciones de personas con discapacidad en la planificación y el proceso de adopción de decisiones para atender a los alumnos y alumnas con necesidades educativas especiales" (UNESCO, 1994, p. ix).

Primeramente, en este artículo realizaré un breve acercamiento a las ONGS/PCDF, su gran diversidad de funciones y finalidades, para luego entrar en los aspectos de la Declaración que siguen vigentes y los que han ido evolucionando, como también reconocer posibles fisuras y dificultades que se filtraron en políticas y prácticas, analizados desde la mirada como ONGS/PCDF. Por último, señalaré algunos desafíos para las ONGS/PCDF para construir una educación y sociedad más inclusivas.

\section{Organizaciones de y para personas con discapacidad y sus familias}

Resulta importante partir de un acercamiento a la identificación de las ONGS/PCDF. Estas organizaciones se pueden haber iniciado con diferentes objetivos: brindar servicios; generar encuentros y apoyos entre semejantes, generar políticas u acciones sociales, etc. A su vez, pueden tratarse de organizaciones referidas a una discapacidad, a varias discapacidades, o a una diversidad de personas; tratarse de organizaciones de familias de personas con discapacidad o de personas con discapacidad, o de ambas; o ser organizaciones no gubernamentales destinadas atender a personas con discapacidad, pero dirigidas por profesionales o por voluntarios, o ambos, las cuales pueden tener, o no, fines de lucro. A su vez, su alcance puede ser local, regional, nacional o internacional. Están también las organizaciones, algunas llamadas federaciones, que nuclean otras asociaciones de acuerdo con distintos criterios, agrupaciones que han logrado un mayor impacto social y político.

Lo fundamental de distinguir en estas organizaciones es su finalidad y acción por una real inclusión, o no, que tiñe sus objetivos generales; si brinda servicios para la inclusión o servicios segregados, o si las acciones y/o políticas que genera están alineadas, o no, al derecho a una educación inclusiva que apela la CDPD. En este aspecto, las ONGS/PCDF son bien disímiles y algunas hasta opuestas en objetivos y finalidades.

Así, décadas atrás, ONGS/PCDF de personas con discapacidad intelectual armaron escuelas especiales para brindar un servicio educativo vedado para estos niños. Con el tiempo, su servicio segregado se constituyó en el fin en sí mismo; y para poder mantenerlo, debieron fortalecerlo, convirtiéndose en un perverso circulo vicioso. Si bien, estas organizaciones 
pueden estar planteadas como sin fines de lucro, terminan priorizando su subsistencia, por sobre los derechos a una inclusión de las personas con discapacidad. Cabe citar a Diaz Velázquez (2008):

\begin{abstract}
Se adopta el carácter prestacional y se cae en lo que Robert Mitchels denominó la "jaula de hierro"; esto es, la organización, cada día más burocratizada, pierde de vista en un primer lugar su fin primogénito y se torna en un fin en sí misma (Mitchels, 1976). No por el hecho de prestar y gestionar servicios para sus asociados, ahora usuarios. Sino porque se convierte en fin instrumental el crecimiento económico y estructural de la entidad. Esto, como ya hemos comentado, las hace más inflexible a los cambios, las convierte en focos de segregación social, principalmente sociolaboral, pues suple de manera "semisegregada" las acciones que los poderes públicos no llevan a cabo, o bien proporcionan un espacio nuevo para el desarrollo de actividades sociales (laborales, educativas, de ocio) en paralelo a los espacios normalizados. Se institucionaliza, quizás, la segregación y la cesión de responsabilidades del Estado a la "sociedad civil" de personas con discapacidad: se establecen cuotas "de corresponsabilidad”, esto es, el Estado financia o cede infraestructuras para que se gestionen externamente. (p. 191)
\end{abstract}

Diaz Velázquez sostiene que el Estado financia estas instituciones segregadas y, por ser subsidiadas, difícilmente dichas organizaciones se pronuncien en contra de sus políticas. Destaca la influencia de las estructuras materiales en los sistemas sociales: "las organizaciones, sobre todo cuanto más estructuradas están, son entidades estáticas, poco flexibles, que reaccionan con dificultades frente a cambios de envergadura" (2008, p. 187).

Algunas ONGS/PCDF han generado servicios prestacionales segregados que cubren desde atención temprana, rehabilitación, escolaridad especial, talleres protegidos, centros de día, hogares protegidos, hasta actividades recreativas segregadas...toda una vida segregada para las personas con discapacidad. Es más, encontramos en Latinoamérica organizaciones que suman a todos estos servicios segregados la atención médica en un gran edificio para las personas con discapacidad, monumento a la discriminación y al inmovilismo.

Así, el cambio hacia una integración y, luego, hacia la inclusión educativa, del paradigma centrado en el déficit o "rehabilitador", al paradigma social, no siempre fue aceptado y promovido por todas las ONGS/PCDF. Unas realizaron una modificación nominal, más que de fondo, sin impacto en las prácticas, aún luego de la sanción de la Declaración y de la CDPD, constituyéndose en barreras importantes.

Otras ONGS/PCDF enarbolaron su defensa de los derechos de las personas con discapacidad desde la Convención y con fuerte impronta combativa o de desarrollo de ideas. Lo positivo es que, en mayor o menor medida, este movimiento asociativo de las ONGS/PCDF, en especial en colaboración en redes, ha tenido un impacto en las políticas y prácticas a nivel educativo, generando conciencia y sensibilización social, presión política ante los gobiernos, relacionándose con medios de difusión y coordinando acciones. De esta manera, algunas ONGS/PCDF fueron logrando la defensa de los derechos de una persona de una familia en concreto o de grupos de personas con discapacidad, como también otras organizaciones han logrado acciones de mayor alcance social a nivel nacional e internacional. Sin duda, pueden enfrentar dificultades en su propio accionar como, por ejemplo, el insuficiente compromiso o participación de algunos de sus miembros, el conformismo de algunas familias o su resistencia a los cambios, el trabajo voluntario no sostenido, la falta de planificación estratégica, los escasos recursos y tantos otros obstáculos. 
En este artículo me referiré a las ONGS/PCDF, fundadas y dirigidas por personas con discapacidad y/o sus familias, sin fines de lucro. Como movimiento asociativo de la sociedad civil, no es un actor único y uniforme, sino complejo y diverso. Se fue generando para atender o defender a las personas con discapacidad, buscando respuestas a sus necesidades concretas, en un aquí y ahora, con proyección futura.

\section{Algunas ideas relevantes de la declaración de Salamanca y su vigencia}

La Declaración enuncia los principios que encarna su espíritu, reflejados en sus disposiciones y recomendaciones, que deben guiar a organizaciones y gobiernos. Marcó un rumbo para las ONGS/PCDF, como también una herramienta clave en la defensa de una inclusión educativa para las personas con discapacidad. Destacaré algunos puntos con plena vigencia, sin pretender ser exhaustiva:

- Enfoque de integración que perfila una inclusión: Si bien la Declaración utiliza el término de "integración", los principios y recomendaciones que incorpora dan cuenta mayormente de un enfoque inclusivo. Hoy la integración se asocia con la adaptación del estudiante a una escuela, un aula y a un programa concebido para la mayoría, siguiendo parámetros homogéneos y rígidos. En cambio, la inclusión implica la transformación profunda del sistema educativo, de las escuelas y las aulas para acoger y enseñar a la diversidad de sus estudiantes; implica identificar barreras para suprimirlas, y asegurar los accesos, apoyos y ajustes para que todos y cada uno puedan aprender juntos, estudiantes con y sin discapacidad. La Declaración asume una postura vanguardista en su enfoque sobre la integración, desarrollando aspectos inclusivos:

$\checkmark$ Derecho a la educación e integración como política educativa: La Declaración reafirmó el derecho a una educación y la necesidad de garantizar una enseñanza de calidad para todos los estudiantes con necesidades educativas especiales (NEE), enmarcada en una Educación Para Todos. Apela a los gobiernos a dar prioridad a la transformación y el mejoramiento de los sistemas educativos para que sean integradores, adoptando leyes, políticas y medidas efectivas que permitan a todos los niños matricularse en escuelas comunes u ordinarias.

$\checkmark$ Atención a la diversidad: La Educación para Todos, no sólo implica integrar a estudiantes con NEE, sino atender a la diversidad. Las escuelas deben enseñar a todos los niños y adolescentes, tenga o no discapacidad, aseverando que "Las escuelas deben acoger a todos los niños independientemente de sus condiciones físicas, intelectuales, sociales, emocionales lingüísticas u otras" (UNESCO, 1994, p. 6); estas tienen el desafío de brindar una educación de calidad para que todos tengan éxito, aún los estudiantes con discapacidades graves. Lo "normal” es que existan diferencias en el aprendizaje, por ello las escuelas deben reconocer diferentes necesidades de sus alumnos y responder a ellas.

Las escuelas integradoras son promotoras de culturas y sociedades inclusivas, por tanto, sus beneficios no son sólo educativos. Así afirma que "las escuelas ordinarias con esta orientación integradora representan el 
medio más eficaz para combatir las actitudes discriminatorias, crear comunidades de acogida, construir una sociedad integradora y lograr la educación para todos" (UNESCO, 1994, p. ix). Toda la comunidad educativa debe comprometerse en el logro de una igualdad de oportunidades y participación, incluso los compañeros.

$\checkmark$ La escuela más cercana: La escuela a la que asista cada estudiante con discapacidad debe ser aquella de su vecindario, la más cercana, "a la escuela a la que debería asistir si no tuviera esa discapacidad” (UNESCO, 1994, p. 17).

$\checkmark$ Aprender juntos en el aula común, más allá de sus dificultades y diferencias, dando la oportunidad que desarrollen entre sí actitudes positivas, reduciendo la discriminación entre los estudiantes.

$\checkmark$ Planes y programas educativos deben ser flexibles y adaptables, así como los procedimientos de evaluación. Propone una Pedagogía Centrada en el Niño que evite la homogeneización de las propuestas de enseñanza que afectan a los estudiantes y a la calidad educativa, cambiando la mentalidad de que "lo que sirve para uno sirve para todos" (UNESCO, 1994, p. 22). Se trata de ajustar el currículo ordinario, ofreciendo opciones curriculares y brindando los apoyos adicionales que los estudiantes con NEE requieran, en vez de seguir un programa de estudios diferente. Remarca la importancia de la evaluación formativa para el seguimiento de los aprendizajes alcanzados.

$\checkmark$ Necesidad de apoyos y programas específicos en el marco de la integración: de atención y educación preescolar; de preparación para la vida adulta para jóvenes con NEE; de alfabetización de adultos con discapacidad; al fomento de la participación de las niñas y mujeres con discapacidad; a desarrollar programas de educación continua para adultos con discapacidades; a contratar a profesores capacitados y a personal de educación con discapacidades, etc.

- Avances en las concepciones de discapacidad y de necesidades educativas especiales: La Declaración les atribuye importancia a las problemáticas del contexto en la gestación de las NEE y de la discapacidad. Además, considera que las NEE no se reducen a los estudiantes con discapacidad, porque muchos niños pueden presentar NEE en algún momento de su escolarización.

Reconoce la Influencia de factores contextuales como generadores de mayor discapacidad: "Durante demasiado tiempo, los problemas de las personas con discapacidades han sido agravados por una sociedad invalidante que se fijaba más en su discapacidad que en su potencial" (UNESCO, 1994, p. 7).

- Responsabilidad de los gobiernos en la formación de los profesionales de las escuelas, desde la formación inicial a la formación continua. Los profesores de las escuelas comunes deben formarse para responder a las necesidades de los estudiantes y trabajar colaborativamente con las familias y los profesionales de apoyo.

La formación especializada en NEE, debe integrarse en la formación común para favorecer la movilidad de los profesionales. Los profesores especializados deberán reexaminar su 
formación según tipo de discapacidad para poder trabajar en diferentes contextos: "Su núcleo común deberá ser un método general que abarque todos los tipos de discapacidades, antes de especializarse en una o varias categorías particulares de discapacidad" (UNESCO, 1994, p. 29). Deberán ofrecer servicios de apoyo colaborativos, coordinado con la escuela, buscando aprovechar los recursos existentes en la comunidad.

- Importancia de la participación de los padres. La educación de niños con NEE debe ser una tarea conjunta entre padres, profesionales, directivos, profesores y administradores, tomando decisiones, supervisando y apoyando al aprendizaje, propiciando la integración escolar y social. Una buena gestión escolar promueve un trabajo colaborativo; propicia una cooperación eficaz entre profesores y profesionales de apoyo y genera programas de investigación y desarrollo institucional. "Cada escuela debe ser una comunidad colectivamente responsable del éxito o el fracaso de cada alumno” (UNESCO, 1994, p. 24).

Como ya mencioné, en la Declaración se destaca que los gobiernos tienen la responsabilidad en la generación de la participación de los padres y de las propias personas con discapacidad, tanto en la planificación como en la toma de decisiones para atender a los alumnos y alumnas con necesidades educativas especiales. (UNESCO, 1994, p. ix). Los padres tienen derecho intrínseco a ser consultados sobre la educación de sus hijos.

- Participación de asociaciones de personas con discapacidad y sus familias: La Declaración insta a los gobiernos a que sean promotores de asociaciones de padres, dándoles apoyo político y legislativo, para que velen por la mejora de la educación de sus hijos:

Se deberá promover la creación de asociaciones de padres y hacer participar a sus representantes en la concepción y aplicación de programas destinados a mejorar la educación de sus hijos. También se deberá consultar a las organizaciones de personas con discapacidades a la hora de diseñar y aplicar los programas. (UNESCO, 1994, p. 38)

Remarca la necesaria consulta a las organizaciones de personas con discapacidad en la formulación e implementación de programas que les atañen. Apela a la intervención de estas organizaciones en la elaboración y evaluación de servicios, que promuevan las trasformaciones necesarias.

- Participación y el apoyo de la comunidad, desde el trabajo en red para promover la integración educativa. La Declaración insta a la participación de la comunidad en la planificación, aplicación y evaluación de una educación integradora para los estudiantes con NEE. Apela a la participación y toma de decisiones con las asociaciones representativas de voluntarios y organizaciones que trabajan en la Rehabilitación Basada en la Comunidad y en la inclusión educativa. Los gobiernos deben apoyar a dichas organizaciones y a la comunidad, para que formulen nuevas ideas y propongan modos innovadores para utilizar los recursos disponibles, como en la creación de escuelas integradoras. Asimismo, llama a la colaboración con universidades y otros organismos en la formación. Este trabajo en red requiere una gestión eficaz:

Para combinar los criterios educativos y sociales sobre las prestaciones educativas especiales se requerirán estructuras de gestión eficaces que favorezcan la cooperación de los distintos servicios en el plano nacional y local y que permitan la colaboración entre las autoridades públicas y los organismos asociativos. (UNESCO, 1994, p. 42) 
La Declaración sostiene la Rehabilitación Basada en la Comunidad para rehabilitar, ofrecer igualdad de oportunidades y facilitar la integración social de las personas con discapacidad. Los servicios de salud deberán coordinarse con los educativos para lograr una gestión y una complementariedad eficaz.

Apela a la sensibilización pública, para que la comunidad tenga una actitud positiva y fomente la integración social de personas con NEE. Los medios de comunicación deberán corregir información errónea y fomentar el optimismo sobre potencial de personas con discapacidad.

- Colaboración y apoyo de la comunidad internacional. La Declaración exhorta a los gobiernos y a la comunidad internacional, a las organizaciones no gubernamentales a velar por el cumplimiento de los principios que sostiene y a colaborar con los gobiernos para implementar una educación integradora; apelamos a la comunidad internacional; en particular instamos a: ... a defender el enfoque de escolarización integradora y apoyar los programas de enseñanza que faciliten la educación de los alumnos y alumnas con necesidades educativas especiales. (UNESCO, 1994, p. x)

Remarca el necesario trabajo en red entre organizaciones gubernamentales y no gubernamentales, regionales e interregionales, las organizaciones internacionales e intergubernamentales, etc. para aunar esfuerzos estratégicamente, para brindar asistencia técnica, aprovechando experiencias, intercambiando información y resultados, para la capacitación de personal, la realización de estudios comparados, la promoción de la creación de asociaciones regionales e internacionales, la difusión, la celebración de reuniones y conferencias regionales, el fomento de iniciativas relacionadas con la Educación para Todos, la investigación, etc.

Estos puntos mencionados, sin ser exhaustivos, mantienen la vigencia de la Declaración y brindaron un fuerte impulso a la participación de las ONGS/PCDF, tanto por la expresa apelación a dicha participación, como por constituirse en una herramienta valiosa para la defensa de una educación inclusiva y de calidad.

\section{Evolución desde la declaración de Salamanca}

La evolución de algunas ideas de la Declaración se observa en la importante sanción de la Convención de los Derechos de las Personas con Discapacidad (ONU, 2006). Argentina la ratifica por Ley 26.378/2008 y la Ley 27.044/2014 le otorga jerarquía constitucional, máxima jerarquía legal. Asimismo, casi todos los países de Latinoamérica han signado y ratificado dicha Convención.

Realizaré aquí, por un lado, un análisis de algunos puntos de la Declaración a la luz de los avances que significó la Convención; avances en la letra y las ideas, que re-focalizaron las finalidades de muchas ONGS/PCDF y fortalecieron su espíritu combativo en defensa de una real inclusión. Como mencioné anteriormente, no todas las organizaciones siguen este camino, sino que algunas continúan ancladas en sus servicios segregados, en la visión integracionista limitante, que les impiden transformar su orientación de modo coherente hacia la inclusión. Asimismo, analizaré aquellas fisuras de la Declaración que su letra dejó entrever, entendidas como pequeñas debilidades que han podido filtrarse, sumados a una 
compleja trama de otros factores políticos, sociales, culturales, educativos, etc.; en políticas integracionistas limitantes y prácticas de integración parcial.

\subsection{La educación inclusiva como enfoque de derechos}

La CDPD abarca todos los derechos de las personas con discapacidad. En este artículo me focalizaré en lo educativo, por ser el aspecto profundizado en la Declaración.

Para la Declaración la integración, si bien la enuncia como un principio, se termina postulando como una estrategia para el logro de una Educación para Todos: "La educación integrada y la rehabilitación apoyada por la comunidad representan dos métodos complementarios de impartir enseñanza a las personas con necesidades especiales" (UNESCO, 1994, p. 17). Si es una estrategia, puede ser adoptada o no. De hecho, postula la opción de la escuela especial (como lo profundizaré más adelante). Allí una fisura para alcanzar la pretendida Educación para Todos, para una inclusión real.

La CDPD reconoce a la inclusión educativa como un derecho de las personas con discapacidad, que debe ser garantizado por los Estados Partes en igualdad de condiciones que los demás. Hoy la Educación Inclusiva se persigue como finalidad formativa para todos y para cada uno, respetando sus derechos. A su vez, la inclusión, es un proceso siempre perfectible, no un resultado acabado. Apunta a construir una sociedad inclusiva, con políticas, culturas y prácticas inclusivas.

\subsection{La concepción sobre discapacidad desde el paradigma social}

La Declaración adopta el término NEE. Si bien asume la influencia del contexto, cuando las define las enmarca en la capacidad o dificultad en el aprendizaje: "En el contexto de este Marco de Acción, el término "necesidades educativas especiales" se refiere a todos los niños y jóvenes cuyas necesidades se derivan de su capacidad o sus dificultades de aprendizaje" (UNESCO, 1994, p. 11). En distintos puntos se refiere expresamente a la discapacidad, aunque no la define como tal. Al colocar la dificultad en los estudiantes, se continúa poniendo el énfasis en el déficit y da pie a otra fisura para la inclusión.

En cambio, la Convención adopta el paradigma social, conceptualizando la discapacidad como construcción social, que deviene de la interacción del sujeto con deficiencias con un entorno que genera barreras a su participación y aprendizajes en igualdad de condiciones que los demás. A nivel general, el contexto social, político, cultural, etc., como a nivel educativo, el contexto escolar o áulico, generan barreras a la participación y al aprendizaje, generando la discapacidad. Resulta así imperioso identificar y minimizar dichas barreras y brindar los accesos, los apoyos y los ajustes que cada uno requiere.

\subsection{La inclusión para todos y cada uno}

La CDPD apunta a la inclusión resueltamente, no a la integración, reafirmando el derecho en una Educación Inclusiva que los gobiernos deben garantizar: "los Estados Parte asegurarán un sistema de educación inclusivo a todos los niveles, así como la enseñanza a lo largo de la vida" (ONU, 2006, Art.24, inc.1). La Convención exige a los Estados Parte garantizar que no se discrimine en la enseñanza a las personas por motivo de discapacidad; es decir, toda forma de separación, segregación o exclusión por motivo de discapacidad es una discriminación.

A la luz de la CDPD y desde una visión crítica, la Declaración relativizó el/la estudiante a integrar "en la medida de lo posible", fortaleciendo la idea y las políticas generadoras de modalidades de integración parcial y discriminatorias, que limitan la real participación y 
el aprendizaje de todas las personas con discapacidad en la escuela y el aula común en igualdad de condiciones. Afirma "La legislación debe reconocer el principio de igualdad de oportunidades de los niños, jóvenes y adultos con discapacidades en la enseñanza primaria, secundaria y superior, enseñanza impartida, en la medida de lo posible, en centros integrados" (UNESCO, 1994, p. 17). A pesar de que la Declaración insta a que los niños con discapacidad asistan a la escuela que hubieran asistido de no tener dicha discapacidad, restringe tal posibilidad al limitarla "en la medida de lo posible".

La Declaración manifiesta que la escolarización en escuelas especiales debe ser una excepción, pero igualmente la sostiene como opción según la situación del niño y la escuela que no satisface sus necesidades:

La escolarización de niños en escuelas especiales - o clases especiales en la escuela con carácter permanente- debiera ser una excepción, que sólo sería recomendable aplicar en aquellos casos, muy poco frecuentes, en los que se demuestre que la educación en las clases ordinarias no puede satisfacer las necesidades educativas o sociales del niño o cuando sea necesario para el bienestar del niño o de los otros niños. (UNESCO, 1994, p. 11)

Marca así otra gran fisura por la que se filtran justificativos para segregar y discriminar a un/a estudiante por sus posibilidades o por una escuela que no satisface sus necesidades (sea porque no lo pretende, no quiere o no sabe hacerlo).

Insta a que sean pocos estudiantes los atendidos en escuelas especiales o aulas especiales: "las escuelas especiales, o los departamentos dentro de las escuelas integradoras, pueden continuar ofreciendo una mejor educación a los relativamente pocos alumnos que no pueden ser atendidos en las escuelas o clases ordinarias" (UNESCO, 1994, p. 12). Además, da pie a modalidades de integración parcial en tanto sostiene que "Incluso en los casos excepcionales en que sea necesario escolarizar a los niños en escuelas especiales, no es necesario que su educación esté completamente aislada. Se deberá procurar que asistan a tiempo parcial a escuelas ordinarias" (UNESCO, 1994, p. 18). Se estaría promoviendo, entonces, la asistencia a la escuela especial o a dos escuelas, especial y común, generando barreras para que ese estudiante participe y aprenda en igualdad de condiciones, sin discriminarlo por motivos de discapacidad, socavando su sentido de pertenencia a un grupo humano diverso e inclusivo en el aula y la escuela común.

Siguiendo esta idea segregacionista, en Argentina la Resolución del Consejo Federal de Educación-RCFE-311/2016 (Art. 12) afirma: "En caso de aquellos niños/as que realicen trayectoria educativa en la Modalidad de Educación Especial, se garantizará como estrategia para la inclusión la implementación de espacios compartidos con escuelas del Nivel que contribuyan a fomentar prácticas inclusivas." Usar el término "prácticas inclusivas" no las convierte en tales; ya que en este caso no lo serían, como lo expresé en el párrafo anterior.

Asimismo, otra interpretación de integrar "en la medida de lo posible" posibilita otras prácticas usuales de integración parcial y limitada, en tanto, incorporar a los/las estudiantes con discapacidad en un aula especial dentro de la escuela común, promoviendo su segregación, generando barreras para su participación plena en igualdad de oportunidades y condiciones. Expresamente la Declaración especifica que los estudiantes con sordera o sordoceguera conviene que se eduquen en clases o escuelas especiales: "Por las necesidades específicas de comunicación de los sordos y los sordos/ciegos, sería más conveniente que se les impartiera una educación en escuelas especiales o en clases y unidades especiales dentro de las escuelas ordinaria" (UNESCO, 1994, p. 18). El déficit del 
sujeto es mal considerado la problemática, cuando es el contexto que debería modificarse para atender a todos. Por tanto, la integración en la Declaración no es realmente para todos, sino para algunos, remarcando la fisura a la inclusión.

Resulta relevante el comentario del Comité de la ONU (2016):

\begin{abstract}
Se debe prohibir que las personas con discapacidad queden excluidas del sistema general de educación mediante, entre otras cosas, disposiciones legislativas o reglamentarias que limiten su inclusión en razón de su deficiencia o grado de dicha deficiencia, condicionando, por ejemplo, la inclusión al alcance del potencial de la persona o alegando una carga desproporcionada o indebida para eludir la obligación de realizar los ajustes razonables. (p. 7)
\end{abstract}

Además, exhorta a "derogar las disposiciones que definen a determinadas categorías de alumnos como, por ejemplo, "ineducables" (ONU, 2016, p. 19).

La expresión "en la medida de lo posible" aparece de modo semejante en la Ley Nacional de Educación de Argentina, en resoluciones nacionales y provinciales. Así afirman que el gobierno "garantizará la integración de los/as alumnos/as con discapacidades en todos los niveles y modalidades según las posibilidades de cada persona” (Ley Nacional de Educación, Art.42), y que la integración se desarrollará "siempre que sea posible" (RCFE 174/2012, Art.15,28,32; RCFE 155/2011, Art.19, 44), limitando la posibilidad de inclusión por la situación de la persona. Estas expresiones continúan mal justificando que a muchos niños/as y adolescentes se le limite en la práctica la inclusión a la escuela común y/o al aula común. Estarían violando la CDPD, por tanto, estas políticas y acciones son anticonstitucionales para nuestro país.

En las Observaciones finales sobre Argentina del Comité de la ONU (2012) expone que existen inconsistencias entre las políticas educativas y las prácticas concretas:

El Comité toma nota de que el marco legislativo que regula la educación en el Estado
parte contiene de manera expresa el principio de la educación inclusiva ... Sin
embargo, observa con preocupación que la implementación de este principio se ve
limitada, en la práctica... El Comité expresa su gran preocupación por el elevado
número de niños y niñas con discapacidad atendidos en escuelas especiales. (p. 6)

Una cuestión relevante respecto a estas restricciones "siempre que sea posible" o "en la medida de lo posible" sería arbitrariamente "establecer" las personas que resuelven quienes son los/las niños/as o adolescentes que estarían en condiciones o no de educarse en las escuelas comunes, acción discriminadora sea que provenga de profesionales, de organismos o de los propios padres. En Argentina, frecuentemente son las escuelas especiales y sus equipos técnicos quienes toman la decisión, generando barreras y discriminación.

El Comité de la ONU (2012) exhorta a Argentina a que tome medidas para garantizar una educación inclusiva de todas las personas con discapacidad y reclama la urgencia que los niños que están escuelas especiales sean incluidos en escuelas comunes:

Urge al Estado parte a tomar las medidas necesarias para que los estudiantes con discapacidad inscritos en escuelas especiales se incorporen a las escuelas inclusivas y a ofrecer ajustes razonables a los estudiantes con discapacidad en el sistema educativo general. (pp. 6-7)

Algunas familias y ONGS/PCDF luchan contra estas injusticias. En ocasiones puede haber una respuesta favorable para la resolución de un caso concreto (Por ejemplo, el ingresar un/a niño/a con discapacidad a la escuela común, o su paso de primaria a secundaria, etc.) pero con relativa influencia en la norma en sí, en su modificación. Unos de los más 
afectados por estas prácticas discriminatorias, son los/as niños/as y jóvenes con discapacidad intelectual, debiendo las ONGS/PCDF enfrentar complejas barreras políticas, sociales, como pedagógicas.

\subsection{Apoyos no enmarcados en escuelas especiales}

Es de destacar que la CDPD no menciona a las escuelas especiales. Según la Declaración, las escuelas especiales deben identificar a los estudiantes con NEE y formar al personal de las escuelas comunes "El personal de estas instituciones especiales posee los conocimientos necesarios para la pronta identificación de los niños con discapacidades. Las escuelas especiales pueden servir también como centros de formación para el personal de las escuelas ordinarias" (UNESCO, 1994, p. 12). Si bien, por un lado, se afirma que los profesores deben desarrollar competencias y autonomía para adaptar los contenidos y metodologías de los programas de estudio a las necesidades individuales de los alumnos y que esta es una tarea de corresponsabilidad, por otro lado, se dice que en esto las escuelas especiales tienen un papel muy importante: "El personal de las escuelas especiales puede aportar una contribución importante a las escuelas ordinarias por lo que respecta a la adaptación del contenido y método de programas de estudios a las necesidades individuales de los alumnos" (UNESCO, 1994, pp. 12-13). Además, se las considera que deben prestar apoyo directo a los niños con NEE:

El apoyo a las escuelas ordinarias podría correr a cargo tanto de las instituciones de formación del profesorado como del personal de extensión de las escuelas especiales. Las escuelas ordinarias deberán utilizar cada vez más estas últimas como centros especializados que prestan apoyo directo a los niños con necesidades educativas especiales. (UNESCO, 1994, p. 31)

Cabe aquí cuestionar, por un lado, el posible rol quizás supletorio del profesor común que generaría la escuela especial y, por otro lado, si tales "apoyos directos" son generadores de segregación del aula común; o si son apoyos fuera de la jornada escolar.

La Declaración insta a la formación de los profesionales de apoyo, no desde cada discapacidad en puntual, sino desde las NEE. Pero el apoyo a una educación inclusiva demanda una formación, no solo desde las NEE, sino desde un enfoque complejo de la diversidad del aula y la escuela, del currículo común flexible y su enseñanza, desde las políticas, culturas y prácticas institucionales, etc. para realizar un apoyo contextual.

Para apoyar la inclusión, la escuela especial debe transformarse profundamente, es decir, dejar de ser "escuela" que enseña a ciertos y determinados estudiantes dentro de sus paredes, discriminados por motivos de la discapacidad, para convertirse en apoyos a las escuelas comunes. Sólo si han logrado este cambio, si se han re-profesionalizado aquellos docentes especiales formados según el déficit de los estudiantes (docentes para estudiantes con sordera, ceguera con discapacidad intelectual, etc.) podrán ser realmente un apoyo para la transformación de las escuelas y aulas comunes en inclusivas, orientado para la enseñanza y el aprendizaje de la diversidad de estudiantes (y no categorías de ellos).

La Declaración promueve la construcción de escuelas integradoras, indicando la no edificación de nuevas escuelas especiales en los países que tengan pocas o ninguna de estas escuelas, por el alto costo que supone. Sin embargo, su posición respecto al rol de las escuelas especiales es quizás una de las fisuras más marcadas y que podrían haberse filtrado en las políticas de Argentina.

Como anticipé, la CDPD no menciona la escuela especial, sin embargo, las políticas nacionales y regionales continúan colocando a las escuelas especiales como las encargadas 
de los procesos de integración: decidiendo que niño/a o adolescente con discapacidad asiste o no a la escuela común, en qué condiciones (inclusiva o por tiempo limitado, o compartido entre la escuela común y la especial, o tiempos en el aula especial dentro de la escuela común), teniendo muchas veces la autoridad de limitar el derecho de todo estudiante con discapacidad a pertenecer y aprender en la escuela y aula común, en el marco de una educación inclusiva donde no se lo discrimine por motivo de su discapacidad.

El Comité de la ONU (2016) exhorta:

Los Estados partes tienen la obligación concreta y permanente de proceder lo más expedita y eficazmente posible para lograr la plena aplicación del artículo 24. Esto no es compatible con el mantenimiento de dos sistemas de enseñanza: un sistema de enseñanza general y un sistema de enseñanza segregada o especial. (p. 13)

La ambigüedad entre las políticas, no conducentes a una real inclusión, y las fisuras señaladas, deben forzar a las ONGS/PCDF a una defensa coherente del derecho a la inclusión, a la real transformación de las escuelas especiales para que no sean barreras para la inclusión; deberán dejar de ser "escuelas".

\subsection{Condiciones equitativas para aprender en el aula común}

Como mencioné, la Declaración insta a una "pedagogía centrada en el niño" para atender las NEE y adaptar los programas de enseñanza. Sin embargo, esto no resulta consistente con otras recomendaciones, por un lado, porque se plantea que los estudiantes con NEE sean incluidos en planes y programas educativos para la mayoría "Cada vez existe un mayor consenso en que los niños y jóvenes con necesidades educativas especiales sean incluidos en los planes educativos elaborados para la mayoría de los niños y niñas" (UNESCO, 1994, p. 6). Por otro lado, porque en la realización de las adaptaciones al currículo tienen un papel importante los profesionales de las escuelas especiales, instituciones organizadas desde del déficit del sujeto, para atender a los estudiantes con NEE. Lo que marca otra fisura para una educación inclusiva y equitativa, donde todos reciban los apoyos que requieren en el aula común según un currículo común flexible y un enfoque contextual.

El Comité de la ONU (2016) apela que los apoyos deben ser inclusivos, en el aula común:

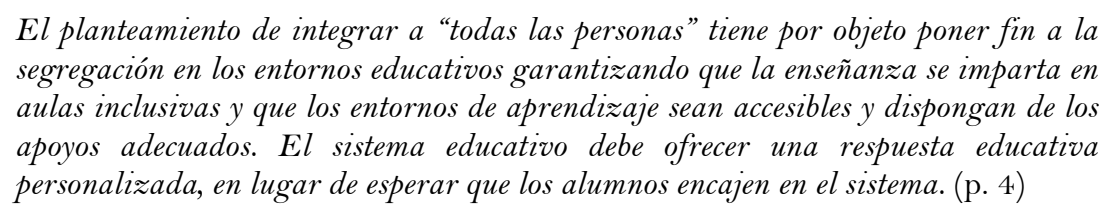

La CDPD exige una educación primaria y secundaria gratuita y de calidad, sin discriminar por motivo de discapacidad, en la comunidad donde viven, prestando los apoyos necesarios y realizando los ajustes en función de las necesidades individuales. Refuerza la necesaria accesibilidad en la educación inclusiva, que debe prestar las ayudas técnicas y necesarias para los/las estudiantes con discapacidad.

La Convención incorpora el concepto de Diseño Universal: "se entenderá el diseño de productos, entornos, programas y servicios que puedan utilizar todas las personas, en la mayor medida posible, sin necesidad de adaptación ni diseño especializado" (ONU, 2006, Art. 2). Aplicado a la enseñanza implica un diseño del currículo común flexible, con alternativas para que todos y cada uno puedan aprender juntos, estudiantes con y sin discapacidad, avanzando según sus ritmos y necesidades, promoviendo la equidad educativa. 
El concepto de Diseño Universal de la Enseñanza (DUE), que hace mayor hincapié en la estructuración de la enseñanza para el aprendizaje de todos, desde un enfoque contextual y holístico. Aplicar el DUE en el aula común inclusiva beneficia a todos los estudiantes, con o sin discapacidad, porque está diseñado para todos y cada uno. (Yadarola, 2016, p. 26)

\subsection{Participación de las familias y de las organizaciones}

La Declaración apela a los gobiernos a promover y facilitar la participación de padres y de las organizaciones de personas con discapacidad y sus familias en la adopción de medidas que le atañen. Sin embargo, hasta las mismas decisiones de los padres estarían enmarcadas en esa expresión "en la medida de lo posible", restringiendo la elección de la escuela: "Los padres son los principales asociados en lo tocante a las necesidades educativas especiales de sus hijos, y a ellos debería corresponder, en la medida de lo posible, la elección del tipo de educación que desean que se imparta a sus hijos" (UNESCO, 1994, pp. 37-38). La medida de lo posible puede "leerse", que la decisión final del tipo de escolaridad (común o especial), la tomarán las "escuelas especiales" o los equipos profesionales, lo que se observa como práctica frecuente en la Argentina. Otra fisura por la cual se filtran políticas y prácticas inadecuadas.

Por ejemplo, la RCFE 311/2016 de Argentina, remarca que los equipos educativos serán los que orientarán y acompañarán las trayectorias escolares de los/as estudiantes con discapacidad, sin incluir dentro de este equipo a la familia en la decisión. Afirma "estos actores, a partir de un trabajo conjunto con la familia y dando lugar a la palabra de el/la estudiante con discapacidad, pondrán en conocimiento las decisiones respecto de las trayectorias escolares..." (Art. 14) Por tanto, "el trabajo conjunto" con las familias es más bien informativo, que decisorio. Además, si las escuelas comunes requieren apoyo en las trayectorias educativas de algún estudiante deberán trabajar con la escuela especial "contar con una propuesta de inclusión elaborada conjuntamente entre los equipos docentes del Nivel y de la Modalidad de Educación Especial, sustentado en el modelo social de la discapacidad" (Art. 7). Se menciona que se trabajará desde el modelo social de la discapacidad, pero esto queda en lo meramente nominal por la discriminación así manifiesta por motivo de la discapacidad. Asimismo, deberán "contar con Proyecto Pedagógico Individual para la Inclusión (PPI)...la planificación y desarrollo del PPI será responsabilidad de los equipos correspondientes, quienes informarán y acordarán con las familias las metas y responsabilidades de cada una de las partes...” Se remarca aquí el papel secundario de las familias en la educación escolar de sus hijos con discapacidad.

La CDPD reconoce el derecho del niño/a y de la persona a la inclusión; no es el derecho de la familia, ni de los profesionales el quitarles ese derecho. El Comité de la ONU (2016) expresa:

La educación inclusiva debe entenderse como... Un derecho humano fundamental de todo alumno. Más concretamente, la educación es un derecho de los alumnos y no de los padres o cuidadores, en el caso de los niños. Las responsabilidades de los padres a este respecto están supeditadas a los derechos del niño. (p. 3)

Además, la Convención apela que las personas con discapacidad deben participar activamente, en especial en los procesos de adopción de decisiones sobre políticas y programas que les atañen directamente. Los Estados Partes deben realizar consultas estrechas y realizar una colaboración activa con las personas con discapacidad y las organizaciones que las representan. El Comité de la ONU (2016) resalta la importancia de esta participación: "Se debe reconocer a las personas con discapacidad y, cuando proceda, 
a sus familias, como colaboradores y no solo beneficiarios de la educación” (p. 2). Muy particularmente, remarca la obligación de los gobiernos de promover dicha participación en las decisiones hacia una educación inclusiva:

Los Estados Partes deberían consultar e integrar activamente a las personas con
discapacidad, incluidos los niños con discapacidad, a través de las organizaciones que
las representan, en todos los aspectos de la planificación, la aplicación, el seguimiento
y la evaluación de políticas y leyes sobre educación inclusiva. (ONU, 2018, p. 18)

Asimismo, la CDPD sostiene la importancia que los Estados Partes promuevan la cooperación de las organizaciones internacionales, regionales, de la sociedad civil y particularmente las organizaciones de personas con discapacidad. Entre las medidas está, velar por la cooperación internacional, para que los programas de desarrollo internacional sean inclusivos y accesibles; intercambio y distribución de información, investigación, asistencia apropiada, etc. La importancia de estas organizaciones también está en el seguimiento de los programas y planes implementados: "La sociedad civil, y en particular las personas con discapacidad y las organizaciones que las representan, estarán integradas y participarán plenamente en todos los niveles del proceso de seguimiento" (ONU, 2006, Art. 33, inc. 3). De esta manera, las ONGS/PCDF tienen una participación importante.

Ahora bien, si para esas ONGS/PCDF llamadas al trabajo cooperativo nacional y/o internacional en defensa a la inclusión son organizaciones que brindan servicios segregados, donde la inclusión está nominal y formalmente incorporada, pero realmente no penetró en sus políticas, culturas y prácticas institucionales de modo coherente, difícilmente su participación sea beneficiosa para encaminar una real inclusión. En el marco de la CDPD, se afirma "El Comité considera que las organizaciones de personas con discapacidad deberían basarse en los principios y derechos reconocidos en la Convención, comprometerse a aplicarlos y respetarlos plenamente (ONU, 2018, p. 3). Sin embargo, ni la Declaración, ni la CDPD ponen como condición que las ONGS/PCDF que participen en tan relevantes decisiones, de impacto local, regional, nacional o internacional, sean inclusivas en políticas y prácticas. Esta falta de explicitación resulta así en una fisura donde se pueden filtrar prácticas no inclusivas.

He señalado, por un lado, la vigencia de la Declaración de Salamanca, marcando grandes avances para la inclusión educativa de las personas con discapacidad, pero, por otro lado, identifique algunas fisuras que han podido filtrarse en Argentina y como en otros países Latinoamericanos, incidiendo aún en políticas y especialmente en prácticas limitantes y discriminatorias, que afectan directamente la vida de las personas con discapacidad. Se hace indispensable trabajar mancomunadamente para encaminar una real inclusión educativa, desde sus políticas, culturas y prácticas, identificando y eliminado fisuras.

\section{Desafíos de las organizaciones de y para personas con discapacidad y sus familias, para lograr sistemas educativos y sociedades más inclusivas}

Lograr sistemas educativos inclusivos, superando viejos esquemas enraizados, no es sencillo. Y los cambios no son siempre ascendentes, sino con avances y retrocesos, con períodos estancados, en definitiva, complejos, porque además inciden gran cantidad de factores entramados. A su vez, lograr sociedades inclusivas, con políticas, culturas y prácticas inclusivas, construir sociedades más justas, equitativas y solidarias, resulta aún 
más complejo. Mencionaré algunos desafíos de las ONGS/PCDF para avanzar en esta conquista:

- Representar y defender el derecho a la inclusión educativa y social, con un compromiso ético con el cambio, con objetivos claros y prácticas coherentes; sin confundir inclusión educativa con integración o segregación. Como afirma el Comité de la ONU: "la integración no garantiza automáticamente la transición de la segregación a la inclusión” (2016, p. 4). Y los gobiernos regionales y organismos internacionales, a la hora de convocar a la participación, deberían convocar a las ONGS/PCDF inclusivas, en sus políticas y en sus prácticas y sin fines de lucro.

- Dar primacía a la defensa y gestación de transformaciones educativa, sociales y políticas, por sobre la oferta de servicios de la ONGS/PCDF, para encaminar la inclusión como proyecto de vida y de sociedad, fortaleciendo la autodeterminación y calidad de vida de las personas con discapacidad.

- Fortalecer y apoyar la participación de personas con discapacidad en las ONGS/PCDF, la autogestión, como el trabajo cooperativo y en red. El Comité de la ONU afirma que las organizaciones de personas con discapacidad "Solo pueden ser aquellas dirigidas, administradas y gobernadas por personas con discapacidad y la mayoría de sus miembros han de ser personas con discapacidad" (2018, p. 3) y que "El papel de los padres, los familiares y los cuidadores en esas organizaciones debería consistir en empoderar y prestar asistencia a las personas con discapacidad para que estas tengan voz y tomen el pleno control de sus vidas" (2018, p. 4).

- Trabajar en redes, tanto a nivel micro (comunidad educativa) como macroeducativo, coordinando entre ONGS/PCDF, otras organizaciones de la sociedad civil, organismos del Estado, organizaciones internacionales, etc., sea en trabajos puntuales o sistemáticos. Promover canales de comunicación abiertos, dinámicos, presenciales, virtuales, diversificados, según contextos y necesidades. El trabajo en red podría favorecer a superar el estatismo y falta de flexibilidad de algunas ONGS/PCDF, que analizamos anteriormente. En esto los organismos internacionales pueden ser claves en orientar rumbos, en colaboración con otros organismos regionales.

- Buscar información relevante y actualizada, experiencias innovadoras, identificar alternativas del contexto y las que se pueden generar que sean inclusivas.

- Formar a las personas con discapacidad y sus familias, a profesionales, a docentes y a directivos y demás miembros de las comunidades educativas, como a otros miembros de la sociedad civil, en los derechos de las personas con discapacidad, en la identificación de barreras y como suprimirlas, en los accesos, ajustes y apoyos necesarios, en estrategias para un trabajo conjunto cooperativo, promoviendo el compromiso ético con la transformación.

- Concientizar y sensibilizar a la sociedad civil, a los medios de comunicación, difundiendo principios y experiencias inclusivas, promoviendo una cultura inclusiva. En este sentido, resulta interesante incorporar el enfoque del llamado 
"Marketing Social”, en tanto el marketing al servicio de la transformación y el mejoramiento social, con sentido ético.

- Exigir la participación activa en la elaboración y en el seguimiento de planes y programas de inclusión educativa y social, a nivel local, regional, nacional e internacional.

- Gestionar las ONGS/PCDF como organizaciones inclusivas e inteligentes, capaces de reflexionar sobre sí mismas y mejorar en consecuencia; realizando un planeamiento estratégico de acciones en función de objetivos inclusivos y alineados a la Convención, encarado desde una mirada proactiva para resolver los problemas que pueden irse presentando a futuro, y no sólo del presente, con acciones estratégicas para obtener y sostener recursos humanos y económicos y con una evaluación continua para la mejora.

Después de estos 25 años jes posible la transformación deseada?

La Declaración de Salamanca significó peldaños fundamentales para progresar hacia una educación y una sociedad inclusiva. Significó una orientación y a su vez, una herramienta clave para las ONGS/PCDF. Y en estos 25 años se fueron dando avances en las políticas (como lo es la CDPD), en culturas y prácticas educativas y sociales, con impacto concreto en la mejora de la vida de las personas con discapacidad y sus familias, de la comunidad.

La inclusión educativa demanda una transformación profunda, compleja y que abarque todos los ámbitos de la sociedad y de la vida de las personas con discapacidad. Por tanto, es una transformación que debe fortalecerse en el tiempo, aunando esfuerzos, sistemáticos y sostenidos, monitoreando cambios y renovando acciones. Pero es posible, como ONGS/PCDF contribuir, en mayor o menor medida, para movilizar transformaciones, quizás con pequeños aportes. Sumando esfuerzos en Redes dichos aportes podrán ser más significativos.

Cabe aquí destacar dos metas de la Agenda 2030 de la ONU (2015): para una educación de calidad "De aquí a 2030, eliminar las disparidades de género en la educación y asegurar el acceso igualitario a todos los niveles de la enseñanza y la formación profesional para las personas vulnerables, incluidas las personas con discapacidad, los pueblos indígenas y los niños en situaciones de vulnerabilidad” (Meta 4.5 ), para la paz, la justicia y las instituciones sólidas "Garantizar la adopción en todos los niveles de decisiones inclusivas, participativas y representativas que respondan a las necesidades" (Meta 16.7). Estas metas, entre muchas otras, fortalecen el rumbo hacia una educación y una sociedad inclusiva y sostenible; a su vez, ponen en "agenda" las acciones para lograrlo, que las ONGS/PCDF debemos acompañar.

Desde nuestra Fundación Síndrome de Down para su Apoyo e Integración (FUSDAI), una ONGS/PCDF fundada en 1990 en Córdoba, Argentina, asumimos el compromiso de promover, antes una real integración, ahora una plena inclusión educativa, laboral y social de las personas con discapacidad, en especial intelectual, y la mejora de su calidad de vida y autodeterminación, buscando ser coherentes entre nuestros objetivos y prácticas concretas, ajustándonos a necesarias transformaciones. Una ONGS/PCDF con reducida infraestructura edilicia, ya que nuestro accionar no está centrado en brindar servicios dentro de nuestras paredes, sino en asesorar, orientar, apoyar, capacitar, sensibilizar, difundir, generar acciones, promover políticas... defender la inclusión en distintos ámbitos de la sociedad civil (escuelas comunes, empresas, organizaciones, gobiernos, sociedad, 
etc.), incluso en países vecinos. Somos conscientes que hemos logrado hacer algunas contribuciones, pero es más la tarea que queda por delante. El trabajo colaborativo y en redes nacionales e internacionales nos da fuerza para continuar en la defensa por un presente y un futuro cada vez más inclusivo, más justo y equitativo.

\section{Referencias}

Diaz Velázquez, E. (2008) El asociacionismo en el ámbito de la discapacidad. Un análisis crítico. Revista Intersticios, 2(2), 183-195.

ONU. (2006). Convención internacional de los derechos de la persona con discapacidad. Recuperado de http://www.un.org/esa/socdev/enable

ONU. (2012). Observaciones finales del comité sobre los derechos de las personas con discapacidad. Comité sobre los derechos de las personas con discapacidad. Recuperado de

https://tbinternet.ohchr.org/_layouts/15/treatybodyexternal/Download.aspx?symbolno $=\mathrm{CRPD} \% 2 \mathrm{fC} \% 2 \mathrm{fARG} \% 2 \mathrm{fCO} \% 2 \mathrm{f} 1 \& \mathrm{Lang}=\mathrm{es}$

ONU. (2015). Agenda 2030 para el desarrollo sostenible. Recuperado de https://www.un.org/sustainabledevelopment/es/2015/09/la-asamblea-general-adoptala-agenda-2030-para-el-desarrollo-sostenible/

ONU. (2016). Convención sobre los derechos de las personas con discapacidad. Comité sobre los derechos de las personas con discapacidad. Observación general $N^{o}$ 4. Artículo 24: Derecho a la educación inclusiva. Recuperado de: https://rededucacioninclusiva.org/situacion-en-laregion/informes-y-documentos/comentario-general-n-4-sobre-el-derecho-a-la-educacioninclusiva/

ONU. (2018). Convención sobre los derechos de las personas con discapacidad. Comité sobre los derechos de las personas con discapacidad. Observación general núm. 7 (2018) sobre la participación de las personas con discapacidad, incluidos los niños y las niñas con discapacidad, a través de las organizaciones que las representan, en la aplicación y el seguimiento de la Convención. Recuperado de: $\quad$ https://confdts 1.unog.ch/1\%20SPA/Tradutek/Derechos_hum_Base/CRPD/oo_Observaciones\%20g enerales\%20CRPD.htm\#GC7

UNESCO. (1994). Declaración de Salamanca. Marco de acción para las necesidades educativas especiales. Recuperado de http://www.unesco.org/education/pdf/SALAMA_S.PDF

Yadarola, M. E. (2016). Diseño universal de la enseñanza para una educación inclusiva. Revista Virtual RedEs, 3, 26-41.

\section{Breve CV de la autora}

\section{$\mathbf{M}^{\mathrm{a}}$ Eugenia Yadarola}

Doctora en Ciencias de la Educación. Presidente (ad-honorem) de la Fundación Síndrome de Down para su Apoyo e Integración -FUSDAI-. Directora de la Línea de Investigación "Inclusión Educativa de Personas con Discapacidad: Políticas y Prácticas”, Universidad Católica de Córdoba -UCC- (como Unidad Asociada al Consejo Nacional de Investigaciones Científicas y Técnicas-CONICET-). Directora de la Carrera de Posgrado de Especialización en Inclusión Educativa de Personas con Discapacidad, UCC. Coordinadora del Programa de Inclusión de la Discapacidad en la UniversidadPROINDU-UCC-. Profesora de grado y posgrado en la UCC-Profesora en el Posgrado 
en la Universidad Nacional de Córdoba. ORCID ID: https://orcid.org/0000-0003-07915716. Email: m.eugenia.yadarola@gmail.com 